\title{
Pessoas com úlceras vasculogênicas em atendimento ambulatorial de enfermagem: estudo das variáveis clínicas e sociodemográficas
}

\author{
PEOPLE WITH VASCULAR ULCERS IN OUTPATIENT NURSING CARE: \\ A STUDY OF SOCIODEMOGRAPHIC AND CLINICAL VARIABLES
}

\author{
PERSONAS CON ÚLCERAS VASCULARES EN ATENCIÓN AMBULATORIA DE \\ ENFERMERÍA: ESTUDIO DE LAS VARIABLES CLIINICAS Y SOCIODEMOGRÁFICAS
}

\section{Suelen Gomes Malaquias', Maria Márcia Bachion², Silvia Maria Soares Carvalho Sant'Ana², Ceres Cristina Bueno Dallarmi ${ }^{4}$, Ruy de Souza Lino Junior ${ }^{5}$, Priscilla Santos Ferreira ${ }^{6}$}

\begin{abstract}
RESUMO
Objetivou-se analisar características sociodemográficas e clínicas de pessoas com úlceras vasculogênicas e investigar a associação entre estas variáveis. Realizou-se um estudo clínico observacional transversal, em salas de curativo de serviços municipais de saúde, de fevereiro a agosto de 2009. Realizou-se entrevista, exame clínico, aplicação da escala Pressure Ulcer Scale Healing (PUSH) e registro fotográfico das lesões. Participaram 42 indivíduos, com média de 60 anos ( \pm 12 ), $73,8 \%$ do sexo masculino, com lesões únicas $(66,7 \%)$, decorrentes de alteração da circulação venosa $(90,5 \%)$, duração de até um ano $(55,5 \%)$. Houve associação entre o escore da PUSH $(\mathrm{p}=0,019)$ e profundidade da lesão $(p=0,027)$ com exercício de atividade ocupacional na atualidade, assim como entre o histórico de tabagismo e o gênero $(p=0,049)$. As características sociodemográficas evidenciadas são semelhantes às de outros, com exceção do gênero masculino, o que indica a necessidade de maiores investigações na população goianiense.
\end{abstract}

\section{DESCRITORES}

Úlcera varicosa

Úlcera da perna

Cicatrização

Cuidados de enfermagem

\begin{abstract}
The aim of this study was to analyze the sociodemographic and clinical characteristics of people with vascular ulcers and to investigate the association between these variables. This cross-sectional, observational clinical study was conducted in outpatient clinics from February to August 2009. Interview, clinical exam, Pressure UIcer Scale Healing and photographic registry of the wounds were performed. Forty-two individuals participated who were, on average, $60( \pm 12)$ years old, $73.8 \%$ males, with single wounds $(66.7 \%)$ resulting from alterations in venous circulation (90.5\%). Their wounds had lasted for up to one year (55.5\%). There was an association between the PUSH score $(p=0.019)$ and depth of wound $(\mathrm{p}=0.027)$ with currently performing occupational activity, as well as between history of tobacco use and gender $(p=0.049)$. The sociodemographic characteristics that were observed were similar to the others, except for being male, which indicates the need for more studies in the population in Goiânia, Brazil.
\end{abstract}

\section{DESCRIPTORS \\ Varicose ulcer \\ Leg ulcer \\ Wound healing \\ Nursing care}

\begin{abstract}
RESUMEN
Se objetivó analizar características sociodemográficas y clínicas de personas con úlceras vasculares e investigar la asociación entre estas variables. Se realizó estudio clínico observacional transversal, en salas de curaciones de servicios municipales de salud, entre febrero y agosto de 2009. Se realizó entrevista, examen clínico, aplicación de escala Pressure Ulcer Scale Healing (PUSH) y registro fotográfico de lesiones. Participaron 42 individuos con promedio de 60 años ( \pm 12 ), 73,8\% de sexo masculino, con lesiones únicas $(66,7 \%)$, derivadas de alteración de la circulación venosa $(90,5 \%)$, duración de hasta un año $(55,5 \%)$. Existió asociación entre el puntaje de PUSH $(\mathrm{p}=0,019)$ y profundidad de la lesión $(p=0,027)$ con ejercicio de actividad ocupacional en la actualidad, así como entre historia de tabaquismo y género $(p=0,049)$. Las características sociodemográficas evidenciadas son semejantes a las de otros, con excepción del género masculino, lo que indica la necesidad de mayores investigaciones en la población goianense.
\end{abstract}

\section{DESCRIPTORES}

Úlcera varicosa

Úlcera de la pierna

Cicatrizacíon de heridas

Atención de enfermería

\footnotetext{
${ }^{1}$ Enfermeira. Mestranda do Programa de Pós-Graduação em Enfermagem da Faculdade de Enfermagem da Universidade Federal de Goiás. Enfermeira da Secretaria Municipal de Saúde de Goiânia. Goiânia, GO, Brasil. sgmalaquias@gmail.com.br ${ }^{2}$ Doutora em Enfermagem. Professora Titular da Faculdade de Enfermagem da Universidade Federal de Goiás. Goiânia, GO, Brasil. mbachion@gmail.com ${ }^{3}$ Enfermeira. Mestranda do Programa de Pós-Graduação em Enfermagem da Faculdade de Enfermagem da Universidade Federal de Goiás. Goiânia, GO, Brasil. silviasoarescarvalho@gmail.com ${ }^{4}$ Graduanda da Faculdade de Medicina da Universidade Federal de Goiás. Goiânia, GO, Brasil. cdallarmi@yahoo.com.br ${ }^{5}$ Enfermeiro. Doutor em Patologia. Professor Adjunto do Instituto de Patologias Tropicais e Saúde Pública da Universidade Federal de Goiás. Goiânia, GO, Brasil. ruy@iptsp.ufg.br 6raduanda da Faculdade de Enfermagem da Universidade Federal de Goiás. Goiânia, GO, Brasil. prisf_enf@yahoo.com.br
} 


\section{INTRODUÇÃO}

Entre as úlceras de perna, as vasculogênicas (de origem venosa, arterial ou mista) são as mais prevalentes, caracterizando-se por um processo crônico, doloroso, recorrente, com impacto negativo na qualidade de vida, na mobilidade, no estado emocional e na capacidade funcional das pessoas acometidas, exigindo atendimento multidisciplinar, com intervenções de natureza local e sistêmica ${ }^{(1-11)}$.

As intervenções de enfermagem a essa população devem considerar múltiplos aspectos entre os quais podem ser citadas as características sociodemográficas tais como gênero, idade, escolaridade, estado civil, renda e ocupação, que irão direcionar a linguagem na abordagem, a preocupação com a necessidade ou não de suporte social e capacidade no envolvimento de ações de prevenção de complicações.

Soma-se a estas, as características de morbidade, tais como o tipo de circulação alterada, o tamanho e a localização da lesão, a existência de lesões anteriores, o estado de cicatrização da lesão e a existência de comorbidades que interferem na circulação e na cicatrização ${ }^{(2-3,10-11)}$.

Estudos realizados com pessoas acometidas por úlceras de perna, em tratamento em unidades de saúde, indicam convergência em algumas características socioeconômicas e demográficas entre a população que apresenta úlceras venosas, tais como, o predomínio do gênero feminino (de 55 a 85\%), baixa renda e escolaridade (em torno de $65 \%)$, e ausência de atividade ocupacional no momento da investigação(1,12-16). Contudo, outras características apresentam-se diversificadas, dependendo da região onde se realizou o estudo. Pesquisas realizadas no Brasil indicam que a faixa etária predominante da população afetada é de $51-70 \operatorname{anos}^{(1,15,17)}$, enquanto que estudos realizados em países da Europa indicam faixa etária predominante de 70 anos ou mais ${ }^{(13,18)}$.

Embora haja consenso, até agora, quanto ao predomínio do gênero feminino ${ }^{(1,12,15-17)}$, foi identificado em uma pesquisa realizada na cidade de Goiânia, maior frequência de pessoas do gênero masculino $(80,4 \%)$ em atendimento à úlceras de perna na atenção básica, com predomínio das úlceras vasculogênicas ${ }^{(17)}$.

Quanto às características de morbidade ${ }^{(1,3,7,13-20)}$, entre as úlceras vasculogênicas, há predomínio da origem venosa (60 a $90 \%$ dos casos). Geralmente as lesões são únicas (variando de 55 a 65\%), sendo a metade distal da perna a mais acometida (até $90 \%$ dos casos), de forma isolada ou não(1-3,12,17-19). A taxa de recorrência é variada, com achados que vão de $38 \%^{(19)}$ a $80 \%^{(1,3,7,20)}$. Em alguns estudos predominam lesões extensas ${ }^{(1,14-15)}$ e em outros leões pequenas ${ }^{(13)}$. O tempo de duração predominante, referido na literatura, varia de meses a anos ${ }^{(1,13)}$.

Assim, é necessário identificar estas características nos diferentes cenários, de forma a compreender as peculiaridades de cada contexto e planejar de forma adequada o atendimento a ser desenvolvido. Usualmente, as pessoas com úlceras vasculogênicas recebem atendimento profissional em unidades básicas de saúde (UBS), nos domicílios, em ambulatórios de serviços de referência, e quando apresentam agravamento das lesões ou complicações de comorbidades, como diabetes mellitus, são atendidas em unidades hospitalares. Apesar do predomínio da rede ambulatorial no atendimento a estas pessoas, ainda há poucos estudos brasileiros realizados no contexto da rede de serviços municipais de saúde, sobre a população acometida por este problema. Compreender melhor este agravo de saúde representa um ponto de partida de ações adequadas da equipe multiprofissional da atenção básica para abordagem a esta clientela, e para política pública de saúde.

\section{Estudos realizados com pessoas acometidas por úlceras de perna,} de saúde em algumas características socioeconômicas e demográficas entre a população que venosas...

\section{OBJETIVOS}

O objetivo geral desta pesquisa foi analisar as características socioeconômicas, demográficas e clínicas de pessoas com úlceras vasculogênicas, entre a população atendida em salas de curativos de unidades municipais de saúde. Os objetivos específicos foram: identificar a presença de úlceras venosas, arteriais ou mistas entre os participantes, descrever a extensão da destruição das camadas da pele (área e características macroscópicas das lesões), caracterizar e investigar a associação entre as características sociodemográficas e de morbidade envolvidas no desenvolvimento de úlceras vasculogênicas entre a população atendida em salas de curativos de unidades municipais de saúde.

\section{MÉTODO}

Trata-se de um estudo clínico descritivo, observacional, transversal, quantitativo, realizado nas salas de curativo de unidades municipais de saúde com funcionamento 24 horas, no período de fevereiro a agosto de 2009. Os critérios de inclusão foram: apresentar úlcera crônica (duração igual ou maior que seis semanas), nos membros inferiores (MMII) em atividade, de origem vascular, em tratamento nas salas de curativos nas unidades envolvidas no estudo. A população constou de 67 indivíduos, sendo que destes 25 foram excluídos, por apresentarem um ou mais dos critérios de exclusão: a) incapacidade de expressar sua aceitação em participar do estudo; b) por falecimento; c) por recusa; ou d) por deixarem de realizar os curativos nas UBS. 
Para a abordagem dos indivíduos, buscavam-se dados sobre a rotina de funcionamento da sala de curativos das unidades de saúde e a existência de usuários que atendiam aos critérios de inclusão no estudo, assim como a assiduidade e horários que costumavam frequentar as unidades. Assim, verificou-se que das 13 unidades municipais de saúde que funcionam 24 horas, 11 prestavam atendimento à pessoas com feridas crônicas, e destas, em dez havia demanda de usuários com úlceras vasculogênicas. Os mesmos foram abordados enquanto aguardavam atendimento. Ao verificar o atendimento aos critérios de inclusão, era apresentado o Termo de Consentimento Livre e Esclarecido (TCLE).

Avaliava-se a disponibilidade de horários dos usuários a cada encontro e, quando necessário, havia novo agendamento para o dia mais próximo em ele que retornasse à unidade. Ocorreram em média três encontros com os usuários, variando de dois a seis, para que fosse completado o protocolo de coleta de dados.

Neste protocolo havia informações relativas aos procedimentos de entrevista, exame clínico, planigrafia digital das úlceras, aplicação da Pressure Ulcer Scale for Healing (PUSH) e a descrição macroscópica da lesão mediante o registro fotográfico das lesões. A entrevista constou de itens para identificação dos aspectos socioeconômicos, demográficos (idade, gênero, classe social, estado civil, anos de estudo, atividade ocupacional que exerceu maior parte da vida e renda pessoal e per capita) e de morbidade (tempo de duração das lesões, número de lesões por participante, recidiva da lesão, zona ou região acometida do membro, índice de massa corporal - IMC, características macroscópicas da lesão, tabagismo atual e anterior) como também questionamentos relacionados aos fatores intervenientes nas alterações vasculares em MMII e na ulceração.

No exame clínico dos MMII, inspecionaram-se alterações pigmentares e/ou tróficas, presença de claudicação, pesquisa de edema, perfusão tissular periférica, temperatura do membro afetado em relação ao contralateral, índice tornozelo-braço (ITB) e antropometria. Se disponível na unidade, realizava-se a consulta ao prontuário. Além disso, registrou-se a localização da lesão, classificando como zona 1 o pé, até os maléolos, zona 2 a metade distal da perna e zona 3 a metade proximal(1).

Para a medida da área das lesões foi realizada a planimetria usando película de acetato e o traçado foi analisado pelo programa Image $\mathrm{J}^{\circledR}(\mathrm{NIH})$, versão $1.38 \mathrm{X}$. A profundidade das lesões foi verificada por dispositivo de ponta romba esterilizado, colocado na região mais profunda do leito da ferida, considerando a linha superficial desta, mediante o posicionamento de régua milimetrada esterilizada, a qual auxiliava na demarcação da altura da ponta do dispositivo.

Para classificação do tipo de alteração circulatória utilizou-se o seguinte conjunto de indicadores clínicos:

- Alteração da circulação venosa - presença de telangectasias e/ou varizes, edema em tornozelo e/ou acima; hiperpigmentação, dermatite ocre, eczema, lipodermatoesclerose; índice tornozelo-braço $\geq 0,9$; exames complementares: mapeamento dupplex indicativo de trombose venosa e/ou insuficiência venosa por obstrução e/ou refluxo dos sistemas venosos.

- Alteração da circulação arterial - claudicação intermitente; dor à elevação do membro afetado; palidez do membro acometido à elevação ou em repouso, pulso(s) diminuído nas artérias da perna; hipotricose; unhas espessadas e/ou irregulares; extremidade fria em relação ao membro não acometido ou área não comprometida com a lesão; índice tornozelo-braço < 0,75; exames complementares: arteriografia ou equivalente indicando obstrução arterial.

- Alteração concomitante da circulação venosa e arterial - presença de indicadores clínicos de alteração tanto venosa como arterial índice tornozelo-braço $>0,5 \leq 0,8$; exames complementares indicativos de alterações venosas e/ou arteriais.

A escala de cicatrização de úlceras por pressão (Pressure Ulcer Scale Healing - PUSH), ferramenta elaborada para avaliar o processo de cicatrização de um tipo específico de lesão, teve seu uso ampliado para feridas crônicas de MMII, e engloba a análise dos parâmetros: área da ferida em $\mathrm{cm}^{2}$, quantidade de exsudato presente na ferida, aparência do leito da ferida. Cada um destes parâmetros possui subescores que somados totalizam entre 0 e 17, sendo que os escores totais maiores indicam piores condições das lesões ${ }^{(21)}$.

A análise dos dados foi auxiliada pelo software Statistical Package of Social Sciences for Windows ${ }^{\circledR}$, versão 16.0. Utilizaram-se procedimentos de estatística descritiva, medidas de tendência central e de dispersão. Para a análise da associação entre as variáveis clínicas (número de lesões por participante, tempo de lesão, escore da PUSH, profundidade das lesões, zona acometida, recidiva, IMC, tabagismo) e das variáveis sociodemográficas (sexo, idade, classe social, anos de estudo, ocupação, atividade exercida, renda pessoal, renda per capita) foi realizada a dicotomização e assim, utilizou-se o teste qui-quadrado ou Exato de Fisher. Foram consideradas estatisticamente significantes diferenças menores ou iguais a $5 \%(p \leq 0,05)$.

A pesquisa foi aprovada pelo Comitê de Ética em Pesquisa da Universidade Federal de Goiás, número de protocolo $032 / 2008$.

\section{RESULTADOS}

A amostra constou de 42 participantes, com média de 60 anos ( \pm 12 ), sendo $73,8 \%$ do gênero masculino, $66,6 \%$ com renda per capita igual ou menor que um salário mínimo, $85,7 \%$ com baixa escolaridade, isto é, indivíduos que referiram ter cursado até o ensino fundamental $(64,3 \%)$ e os que não tiveram nenhum ano de estudo (21,4\%) (Tabela 1$)$.
Pessoas com úlceras vasculogênicas em atendimento ambulatorial de enfermagem: estudo das variáveis clínicas e sociodemográficas Malaquias SG, Bachion MM, Sant'Ana SMSC, Dallarmi CCB, Lino Junior RS, Ferreira PS 
Tabela 1 - Distribuição dos participantes do estudo $(\mathrm{N}=42)$ segundo aspectos sociodemográficos - Unidades de Saúde da Secretaria Municipal de Goiânia, GO - 2009

\begin{tabular}{|c|c|c|}
\hline Variáveis Sóciodemográficas & $\mathbf{F}$ & $\%$ \\
\hline \multicolumn{3}{|l|}{ Gênero } \\
\hline Masculino & 31 & 73,8 \\
\hline Feminino & 11 & 26,2 \\
\hline \multicolumn{3}{|l|}{ Idade } \\
\hline$<40$ & 7 & 16,7 \\
\hline $501-60$ & 14 & 33,3 \\
\hline $601-70$ & 12 & 28,6 \\
\hline 70 ou mais & 9 & 21,4 \\
\hline \multicolumn{3}{|l|}{ Classe social* } \\
\hline $\mathrm{B} 1$ e B2 & 11 & 26,3 \\
\hline $\mathrm{C} 1$ e $\mathrm{C} 2$ & 13 & 30,9 \\
\hline $\mathrm{D}$ & 13 & 30,9 \\
\hline $\mathrm{E}$ & 5 & 11,9 \\
\hline \multicolumn{3}{|l|}{ Estado Civil } \\
\hline Casados, união consensual & 22 & 52,4 \\
\hline Divorciados, separados, solteiros ou viúvos & 20 & 47,6 \\
\hline \multicolumn{3}{|l|}{ Anos de estudo } \\
\hline Nenhum & 09 & 21,4 \\
\hline Até 5 anos & 12 & 28,6 \\
\hline 6 a 10 anos & 12 & 28,6 \\
\hline Mais de 10 anos & 09 & 21,4 \\
\hline \multicolumn{3}{|l|}{ Exercício de atividade ocupacional } \\
\hline Não está em atividade & 29 & 69,1 \\
\hline Está em atividade & 13 & 30,9 \\
\hline \multicolumn{3}{|l|}{ Atividade que exerceu maior parte da vida } \\
\hline Construção civil & 08 & 19,0 \\
\hline Serviços rurais (lavrador) & 06 & 14,3 \\
\hline Motorista & 05 & 11,9 \\
\hline Vigilante & 05 & 11,9 \\
\hline Do lar & 04 & 9,5 \\
\hline Costureira & 05 & 11,9 \\
\hline Outros & 09 & 21,5 \\
\hline \multicolumn{3}{|l|}{ Renda } \\
\hline \multicolumn{3}{|l|}{ Renda pessoal } \\
\hline$\leq 1$ salário mínimo & 25 & 59,5 \\
\hline$>1$ salário mínimo & 17 & 40,5 \\
\hline \multicolumn{3}{|l|}{ Renda per capita } \\
\hline$\leq 1$ salário mínimo & 28 & 66,6 \\
\hline$>1$ salário mínimo & 14 & 33,4 \\
\hline
\end{tabular}

* Critério de Classificação Econômica Brasil da Associação Brasileira de Empresas de Pesquisa (CCEB/ ABEP).

Trinta e oito $(90,5 \%)$ apresentaram ITB igual ou maior a 0,9 e características de comprometimento venoso. Observaram-se características de alterações venosas como varizes e/ou telangectasias em todos os participantes (100\%), edema em 40 (95,2\%), dermatite ocre em 25 (59,5\%) e lipodermatoesclerose em 17 (40,5\%) participantes.
O número total de úlceras apresentadas pelos participantes foi 63 lesões. Vinte e oito $(66,7 \%)$ participantes apresentavam uma única lesão, sendo que 35 (55,5\%) das lesões apresentaram tempo da duração menor que um ano (Tabela 2). 
Tabela 2 - Distribuição das feridas $(\mathrm{N}=63)$ segundo variáveis de morbidade dos participantes $(\mathrm{N}=42)$ - Unidades de Saúde da Secretaria Municipal de Goiânia, GO - 2009

\begin{tabular}{|c|c|c|c|c|}
\hline Variáveis de morbidade & Valores & & $\mathbf{f}$ & $\%$ \\
\hline \multirow{5}{*}{$\begin{array}{l}\text { Tempo de duração das } \\
\text { lesões }(\mathrm{N}=63)\end{array}$} & \multicolumn{2}{|l|}{$\leq 1$ ano } & 35 & 55,5 \\
\hline & \multicolumn{2}{|l|}{$>1$ ano $\mathrm{a} \leq 5$ anos } & 14 & 22,2 \\
\hline & \multicolumn{2}{|l|}{$>5$ anos $\mathrm{a} \leq 15$ anos } & 03 & 4,8 \\
\hline & \multicolumn{2}{|l|}{$>15$ anos } & 03 & 4,8 \\
\hline & \multicolumn{2}{|l|}{ Lesões de participantes que não sabiam responder } & 08 & 12,7 \\
\hline \multirow{4}{*}{$\begin{array}{l}\text { Numero de lesões por } \\
\text { participante }(\mathrm{N}=63)\end{array}$} & \multicolumn{2}{|l|}{1 lesão } & 28 & 66,7 \\
\hline & \multicolumn{2}{|l|}{2 lesões } & 09 & 21,4 \\
\hline & \multicolumn{2}{|l|}{3 lesões } & 03 & 7,1 \\
\hline & \multicolumn{2}{|l|}{4 lesões } & 02 & 4,8 \\
\hline \multirow[t]{2}{*}{ Recidiva da lesão $(\mathrm{N}=42)$} & \multicolumn{2}{|l|}{ Sim } & 17 & 40,5 \\
\hline & \multicolumn{2}{|l|}{ Não } & 25 & 59,5 \\
\hline \multirow[t]{2}{*}{ Zona 2 acometida $(\mathrm{N}=42)$} & \multicolumn{2}{|l|}{ Sim } & 39 & 92,9 \\
\hline & \multicolumn{2}{|l|}{ Não } & 03 & 7,1 \\
\hline \multirow[t]{18}{*}{ PUSH } & \multirow{5}{*}{$\begin{array}{l}\text { Área das lesões em } \mathrm{cm} 2 / \text { subescore } \\
(\mathrm{N}=63)\end{array}$} & $\leq 1 / \leq 3$ & 02 & 3,2 \\
\hline & & $>1 \mathrm{a} \leq 8 / 4-7$ & 22 & 34,9 \\
\hline & & $<8$ a $\leq 12 / 8$ & 06 & 9,5 \\
\hline & & $<12 \mathrm{a} \leq 24 / 9$ & 10 & 15,9 \\
\hline & & $>24 / 10$ & 23 & 36,5 \\
\hline & \multirow{4}{*}{$\begin{array}{l}\text { Pior subescore de quantidade de exsudato } \\
(\mathrm{N}=42)\end{array}$} & Ausente & 03 & 7,1 \\
\hline & & Pequena & 07 & 16,7 \\
\hline & & Moderada & 14 & 33,3 \\
\hline & & Grande & 18 & 42,9 \\
\hline & \multirow[t]{5}{*}{ Pior subescore de tipo de tecido $(\mathrm{N}=42)$} & Fechado & - & - \\
\hline & & Tecido epitelial & - & - \\
\hline & & Tecido de granulação & 07 & 16,7 \\
\hline & & Esfacelo & 30 & 71,4 \\
\hline & & Escara & 05 & 11,9 \\
\hline & \multirow[t]{4}{*}{ Escore total das lesões $(\mathrm{N}=63)$} & $\leq 6$ & 03 & 4,8 \\
\hline & & $>6 \mathrm{a} \leq 10$ & 13 & 20,6 \\
\hline & & $>10 \mathrm{a} \leq 15$ & 25 & 39,7 \\
\hline & & $>15$ a 17 & 22 & 34,9 \\
\hline \multirow{2}{*}{$\begin{array}{l}\text { Profundidade em } \mathrm{cm} \\
(\mathrm{N}=63)\end{array}$} & \multicolumn{2}{|l|}{$\leq 0,5$} & 41 & 65,1 \\
\hline & $>0,5$ & & 22 & 34,9 \\
\hline Variáveis de morbidade & Valores & & f & $\%$ \\
\hline Percentual tecidos no & Escara & Sem Escara & 53 & 84,1 \\
\hline leito das lesões $(\mathbf{N}=63)$ & & $<25 \%$ & 08 & 12,7 \\
\hline & & $25 \% \mathrm{a}<50 \%$ & 02 & 3,2 \\
\hline & & $50 \% \mathrm{a}<75 \%$ & - & - \\
\hline & & $>75 \%$ & - & - \\
\hline & Esfacelo & Sem Esfacelo & 07 & 11,1 \\
\hline & & $<25 \%$ & 21 & 33,3 \\
\hline & & $25 \% \mathrm{a}<50 \%$ & 08 & 12,7 \\
\hline & & $50 \% \mathrm{a}<75 \%$ & 13 & 20,6 \\
\hline & & $>75 \%$ & 14 & 22,3 \\
\hline
\end{tabular}


..continuação

\begin{tabular}{|c|c|c|c|c|}
\hline Variáveis de morbidade & Valores & & $\mathbf{f}$ & $\%$ \\
\hline \multirow{5}{*}{$\begin{array}{l}\text { Percentual tecidos no } \\
\text { leito das lesões }(\mathrm{N}=63)\end{array}$} & Tecido de granulação & Sem Tecido de granulação & 02 & 3,2 \\
\hline & & $<25 \%$ & 12 & 19,0 \\
\hline & & $25 \% \mathrm{a}<50 \%$ & 06 & 9,5 \\
\hline & & $50 \% \mathrm{a}<75 \%$ & 14 & 22,3 \\
\hline & & $>75 \%$ & 29 & 46,0 \\
\hline \multirow[t]{2}{*}{ IMC $(N=42)$} & Normal ou baixo peso & & 22 & 47,6 \\
\hline & Sobrepeso ou obesidade & & 20 & 52,4 \\
\hline \multirow{2}{*}{$\begin{array}{l}\text { Histórico de tabagismo } \\
(\mathrm{N}=42)\end{array}$} & Sim & & 30 & 71,4 \\
\hline & Não & & 12 & 28,6 \\
\hline \multirow[t]{2}{*}{ Tabagismo atual } & Sim & & 08 & 19,0 \\
\hline & Não & & 34 & 81,0 \\
\hline
\end{tabular}

Um participante $(2,4 \%)$ apresentou ITB igual a 0,73 no membro afetado e ITB 0,4 em membro contralateral, claudicação intermitente, palidez à elevação dos membros inferiores em menos de 40 segundos, pulsos diminuídos, hipotricose em região perilesional, unhas espessadas e irregulares, bordas circunscritas, com necrose em aproximadamente $60 \%$ do leito da lesão, exsudato em pequena quantidade, sendo este caso classificado como úlcera arterial.

Três pessoas $(7,1 \%)$ apresentavam valores do ITB entre 0,5 e $\leq 0,8$ como também características de alteração venosa (edema, dermatite ocre, lipodermatoesclerose, hiperqueratose e/ou eritema), fazendo supor tratar-se de um caso de úlcera de origem mista.
Quanto a localização das úlceras, verificou-se que a área mais acometida foi a zona 2, de modo isolado em $31(49,2 \%)$ das lesões e conjuntamente à zona 1 em 19 $(30,1 \%)$ casos. Verificaram-se entre as sessenta e três lesões analisadas, predomínio de tecido de granulação $(46,0 \%)$ em quantidade superior a $75 \%$ no leito das feridas, e esfacelo entremeado ao tecido de granulação em $88,9 \%$ das feridas. A maioria $(84,1 \%)$ das lesões não apresentava escara (Tabela 2).

Aproximadamente metade dos participantes apresentou sobrepeso ou obesidade. Os participantes apresentavam histórico de tabagismo em $71,4 \%$ dos casos e na época da avaliação realizada no estudo esta prática havia diminuído drasticamente, uma vez que era presente para apenas 19,0\%.

Tabela 3 - Associação entre variáveis clínicas e sociodemográficas dos participantes. Unidades de Saúde da Secretaria Municipal de Goiânia, GO - 2009

\begin{tabular}{|c|c|c|c|c|c|c|c|c|c|c|c|c|c|c|c|c|c|c|c|}
\hline \multirow[t]{2}{*}{ Variáveis } & & \multicolumn{2}{|c|}{$\begin{array}{l}\text { Número de } \\
\text { lesões }\end{array}$} & \multirow[t]{2}{*}{$\mathbf{p}$} & \multicolumn{2}{|c|}{$\begin{array}{c}\text { Tempo das } \\
\text { lesões em } \\
\text { anos }\end{array}$} & \multirow[t]{2}{*}{$\mathbf{p}$} & \multicolumn{2}{|c|}{$\begin{array}{l}\text { Escore da } \\
\text { PUSH }\end{array}$} & \multirow[t]{2}{*}{$\mathbf{p}$} & \multicolumn{2}{|c|}{$\begin{array}{l}\text { Profundidade } \\
\text { em cm }\end{array}$} & \multirow[t]{2}{*}{$\mathbf{p}$} & \multicolumn{2}{|c|}{$\begin{array}{l}\text { Zona } 2 \text { aco- } \\
\text { metida }\end{array}$} & \multirow[t]{2}{*}{$\mathbf{p}$} & \multicolumn{2}{|c|}{ Recidiva } & \multirow[t]{2}{*}{$\mathbf{p}$} \\
\hline & & 1 & $>1$ & & $\leq 1$ & $>1$ & & $\leq 14$ & $>14$ & & $\leq \mathbf{0 , 5}$ & $>0,5$ & & sim & não & & $\operatorname{sim}$ & não & \\
\hline \multirow{2}{*}{ Sexo } & M & 20 & 11 & $0,723^{\mathrm{a}}$ & 20 & 11 & $0,723^{\mathrm{a}}$ & 14 & 17 & $0,292^{\mathrm{b}}$ & 17 & 14 & $0,477^{\mathrm{a}}$ & 30 & 01 & $0,163^{\mathrm{a}}$ & 11 & 20 & $0,305^{a}$ \\
\hline & $\mathrm{F}$ & 08 & 03 & & 08 & 03 & & 07 & 04 & & 08 & 03 & & 09 & 02 & & 06 & 05 & \\
\hline \multirow{2}{*}{ Idade } & $<60$ & 12 & 09 & $0,190^{\mathrm{b}}$ & 13 & 08 & $0,513^{b}$ & 10 & 11 & $0,758^{b}$ & 14 & 07 & $0,346^{\mathrm{b}}$ & 20 & 01 & $1,000^{\mathrm{a}}$ & 08 & 13 & $0,753^{b}$ \\
\hline & $\geq 60$ & 16 & 05 & & 15 & 06 & & 11 & 10 & & 11 & 10 & & 19 & 02 & & 09 & 12 & \\
\hline \multirow{2}{*}{ Classe social } & $\mathrm{A}, \mathrm{B}, \mathrm{C}$ & 17 & 07 & $0,508^{\mathrm{b}}$ & 17 & 07 & $0,508^{\mathrm{b}}$ & 12 & 12 & $1,000^{\mathrm{b}}$ & 17 & 07 & $0,085^{\mathrm{b}}$ & 22 & 02 & $1,000^{\mathrm{a}}$ & 09 & 15 & $0,650^{\mathrm{b}}$ \\
\hline & $\mathrm{D}, \mathrm{E}$ & 11 & 07 & & 11 & 07 & & 09 & 09 & & 08 & 10 & & 17 & 01 & & 08 & 10 & \\
\hline \multirow{2}{*}{$\begin{array}{l}\text { Anos de } \\
\text { estudo }\end{array}$} & $\leq 4$ & 19 & 07 & $0,261^{b}$ & 18 & 08 & $0,653^{\mathrm{b}}$ & 16 & 10 & $0,057^{\mathrm{b}}$ & 16 & 10 & $0,735^{\mathrm{b}}$ & 25 & 01 & $0,547^{\mathrm{a}}$ & 10 & 16 & $0,735^{\mathrm{b}}$ \\
\hline & $>4$ & 09 & 07 & & 10 & 06 & & 05 & 11 & & 09 & 07 & & 14 & 02 & & 07 & 09 & \\
\hline \multirow{2}{*}{$\begin{array}{l}\text { Exercício de } \\
\text { atividade ocupacional }\end{array}$} & Sim & 08 & 05 & $0,729^{\mathrm{a}}$ & 10 & 03 & $0,486^{\mathrm{a}}$ & 10 & 03 & $0,019^{b}$ & 11 & 02 & $0,027^{\mathrm{a}}$ & 12 & 01 & $1,000^{\mathrm{a}}$ & 05 & 08 & $0,859^{b}$ \\
\hline & Não & 20 & 09 & & 18 & 11 & & 11 & 18 & & 14 & 15 & & 27 & 02 & & 08 & 17 & \\
\hline \multirow{2}{*}{$\begin{array}{l}\text { Ortostatismo na } \\
\text { atividade exercida }\end{array}$} & Sim & 20 & 11 & $0,723^{\mathrm{a}}$ & 21 & 10 & $1,000^{\mathrm{a}}$ & 14 & 17 & $0,292^{\mathrm{b}}$ & 17 & 14 & $0,477^{\mathrm{a}}$ & 30 & 01 & $0,163^{\mathrm{a}}$ & 12 & 19 & $0,733^{a}$ \\
\hline & Não & 08 & 03 & & 07 & 04 & & 07 & 04 & & 08 & 03 & & 09 & 02 & & 05 & 06 & \\
\hline \multirow{2}{*}{$\begin{array}{l}\text { Renda } \\
\text { pessoal }\end{array}$} & $\leq 1 \mathrm{sm}$ & 17 & 08 & $1,000^{\mathrm{a}}$ & 15 & 10 & $0,266^{\mathrm{b}}$ & 11 & 14 & $0,346^{\mathrm{b}}$ & 14 & 11 & $0,573^{\mathrm{b}}$ & 23 & 02 & $1,000^{\mathrm{a}}$ & 10 & 15 & $0,939^{b}$ \\
\hline & $>1 \mathrm{sm}$ & 11 & 06 & & 13 & 04 & & 10 & 07 & & 11 & 06 & & 16 & 01 & & 07 & 10 & \\
\hline \multirow{2}{*}{$\begin{array}{l}\text { Renda per } \\
\text { capita }\end{array}$} & $\leq 1 \mathrm{sm}$ & 19 & 09 & $1,000^{\mathrm{a}}$ & 19 & 09 & $1,000^{\mathrm{b}}$ & 13 & 15 & $0,513^{b}$ & 17 & 11 & $0,824^{\mathrm{b}}$ & 27 & 01 & $0,254^{\mathrm{a}}$ & 12 & 16 & $0,657^{\mathrm{b}}$ \\
\hline & $>1 \mathrm{sm}$ & 09 & 05 & & 09 & 05 & & 08 & 06 & & 08 & 06 & & 12 & 02 & & 05 & 09 & \\
\hline
\end{tabular}

a- Exato de Fisher; b- Qui-quadrado 
Verificou-se associação significativa entre o escore da PUSH $(p=0,019)$, a profundidade máxima da lesão $(p=0,027)$, e a presença de atividade laboral atual, sendo que na presença de escores mais altos da PUSH, e feridas mais profundas os participantes não exerciam atividades laborais no presente (Tabela 3).
O histórico de tabagismo mostrou-se mais frequente no gênero masculino $(p=0,049)$ como mostra a tabela 4 , não havendo associação com qualquer outra variável sócio-econômica, demográfica ou clínica (Tabela 4).

Tabela 4 - Associação entre histórico de tabagismo e Índice de Massa Corporal (IMC) com variáveis clínicas e sociodemográficas. Unidades de Saúde da Secretaria Municipal de Goiânia, GO - 2009

\begin{tabular}{|c|c|c|c|c|c|c|c|}
\hline \multirow[t]{2}{*}{ Variáveis } & & \multicolumn{2}{|c|}{ Histórico de tabagismo } & \multirow[t]{2}{*}{$\mathbf{P}$} & \multicolumn{2}{|c|}{$\begin{array}{c}\text { IMC indicativo de } \\
\text { sobrepeso ou obesidade }\end{array}$} & \multirow[t]{2}{*}{$\mathbf{P}$} \\
\hline & & $\operatorname{sim}$ & não & & $\operatorname{sim}$ & não & \\
\hline \multirow[t]{2}{*}{ Gênero } & M & 25 & 06 & $0,049^{\mathrm{a}}$ & 16 & 15 & $0,384^{\mathrm{a}}$ \\
\hline & $\mathrm{F}$ & 05 & 06 & & 04 & 07 & \\
\hline \multirow[t]{2}{*}{ Idade } & $<60$ & 16 & 05 & $0,495^{b}$ & 09 & 12 & $0,537^{b}$ \\
\hline & $\geq 60$ & 14 & 07 & & 11 & 10 & \\
\hline \multirow[t]{2}{*}{ Escore PUSH } & $\leq 14$ & 16 & 05 & $0,495^{\mathrm{b}}$ & 09 & 12 & $0,537^{\mathrm{b}}$ \\
\hline & $>14$ & 14 & 07 & & 11 & 10 & \\
\hline \multirow{3}{*}{$\begin{array}{l}\text { Tempo maior entre as } \\
\text { lesões apresentadas pelos } \\
\text { participantes }\end{array}$} & & & & & & & \\
\hline & $\leq 1$ ano & 20 & 08 & $1,000^{\mathrm{b}}$ & 06 & 14 & $0,662^{b}$ \\
\hline & $>1$ ano & 10 & 04 & & 08 & 14 & \\
\hline \multirow[t]{2}{*}{$\begin{array}{l}\text { Tipo de circulação } \\
\text { comprometida }\end{array}$} & Venosa & 26 & 12 & $0,308^{\mathrm{a}}$ & 21 & 17 & $0,333^{\mathrm{a}}$ \\
\hline & Arterial ou mista & 04 & 00 & & 01 & 03 & \\
\hline \multirow[t]{2}{*}{ Recidiva } & Sim & 13 & 04 & $0,731^{\mathrm{a}}$ & 10 & 10 & $0,231^{\mathrm{b}}$ \\
\hline & Não & 17 & 08 & & 07 & 15 & \\
\hline
\end{tabular}

a- Exato de Fisher; b- Qui-quadrado

\section{DISCUSSÃO}

No presente estudo, observou-se predomínio de participantes do gênero masculino, assemelhando-se a outro realizado no mesmo cenário de atenção básica em Goiânia, no qual foi encontrado percentual próximo a $70 \%$ de prevalência do gênero masculino, entre pessoas com úlceras de perna, destacando-se a etiologia vascular como a predominante ${ }^{(17)}$. Em contrapartida, nos estudos em outros cenários tem sido encontrado predomínio do sexo feminino ${ }^{(1,13)}$, chegando a $80 \%^{(14)}$.

Este achado necessita ser mais bem estudado, porém vislumbram-se algumas suposições que podem ocorrer no cenário pesquisado, como a possibilidade de que as mulheres realizem tratamento predominantemente no domicílio, ou ainda os homens podem estar suscetíveis à fatores de risco que são mais prevalentes no local de estudo, necessitando de abordagem que considere suas especificidades.

Um estudo(20) chamou a atenção para o fato de que, supor que as úlceras vasculogênicas sejam uma ocorrência caracteristicamente do sexo feminino, com idade maior que 60 anos, pode ser um equívoco, uma vez que a insuficiência venosa ocorre em aproximadamente $30 \%$ da população adulta, sendo as varizes no homem mais calibrosas que nas mulheres. Foi identificado nesta pesquisa que, entre pessoas que apresentaram úlcera venosa antes dos 50 anos predominou o sexo masculino $(59,0 \%)$, enquanto que na população acometida por este agravo após os 50 anos predominou o sexo feminino (77\%) $(p=0,001)$.

A idade dos participantes foi semelhante a um estudo realizado numa cidade da região nordeste ${ }^{(17)}$, porém, menor que a de outros estudos ${ }^{(1,9,13-14,16)}$, mas sem indicar alteração substancial na faixa etária da população predominante acometida, que é acima dos 50 anos.

A renda precária e baixa escolaridade, encontradas na amostra estudada, é uma constante nos estudos que incluem pessoas com úlceras venosas ${ }^{(1,9,13-14,16)}$, podendo indicar estilo de vida que favoreça o aparecimento de lesões ou ainda a falta de acesso a serviços de saúde especializado ou manejo inadequado por parte dos profissionais que prestam atendimento à esta população.

Esta situação pode comprometer a adesão ao tratamento, que apresenta custo expressivo, tanto para o usuário do serviço como para a instituição de saúde ${ }^{(3)}$.

Por outro lado, no sistema público de saúde há demanda reprimida nas áreas de especialidade como a angiologia, fazendo com que esta avaliação bem como a abordagem sistêmica ocorra num momento em que as lesões já estão francamente instaladas.
Pessoas com úlceras vasculogênicas em atendimento ambulatorial de enfermagem: estudo das variáveis clínicas e sociodemográficas Malaquias SG, Bachion MM, Sant'Ana SMSC, Dallarmi CCB, Lino Junior RS, Ferreira PS 
O predomínio de lesões únicas, observado entre os participantes do presente estudo, também é encontrado nas pesquisas de modo geral ${ }^{(1,14,17)}$. Isso pode representar um fenômeno decorrente do longos períodos de curso da lesão, que possibilitou a confluência de lesões múltiplas, ou ainda menor gravidade da alteração vasculogênica.

Quanto a duração das lesões, observam-se variações tanto neste estudo como nas informações trazidas pela literatura ${ }^{(15,17)}$, o que reforça a interação entre fatores como: a gravidade da alteração vasculogênica, o acesso a serviços de atendimento na área de angiologia, o atendimento por uma equipe qualificada, o estilo de vida e adesão ao tratamento e ao serviço.

Os índices de recorrência das feridas, encontrados neste estudo, se assemelham a outro ${ }^{(19)}$, porém se distanciam dos achados da maioria dos estudos ${ }^{(1,7,14)}$. Este fato merece maiores investigações em diferentes cenários, pois há possibilidade de que unidades de referência, como algumas das que participaram dos estudos mencionados, atendendam uma clientela com lesões mais complexas que as usualmente encontradas em unidades de saúde municipais.

Quanto ao tipo de circulação vascular comprometida, a ocorrência de lesões decorrentes de alteração da circulação venosa mantém-se em destaque em relação às demais, semelhantemente a muitos estudos ${ }^{(1,13-20)}$, o que aponta, mais uma vez, para a necessidade de uma abordagem sistêmica e multidisciplinar, que inclua acesso a serviço de angiologia.

Observou-se que os participantes que estavam em atividade na época da avaliação realizada nesta pesquisa apresentaram profundidade da ferida e escore da PUSH menores que aqueles participantes que não estavam em atividade ocupacional no período de coleta de dados. Isto pode decorrer do fato de que para estes indivíduos a lesão pode estar comprometendo a capacidade para exercer alguma atividade. Pesquisas indicam que as úlceras, especialmente venosas, comprometem a capacidade funcional das pessoas com este agravo, bem como interferem na sua mobilidade, além de provocar dor ${ }^{(4,13)}$. Isto pode interferir na capacidade de trabalho das pessoas, desencadeando uma série de consequências com repercussão sobre os aspectos socioeconômicos.

Cabe ao enfermeiro importante papel na articulação de uma forma de organização do processo de trabalho pa-

\section{REFERÊNCIAS}

1. Frade MAC, Cursi IB, Andrade FF, Soares SC, Ribeiro WS, Santos SV, et al. Úlcera de perna: um estudo de casos em Juiz de Fora - MG (Brasil) e região. An Bras Derm. 2005;80(1):41-6. ra o atendimento às necessidades dos usuários, integrando as atividades de cuidar com a qualificação e competência necessária, aos processos gerenciais e relacionais ${ }^{(22)}$.

Este estudo aponta para algumas importantes implicações à prática de enfermagem, a saber:

- reflexão sobre a sua participação no desenvolvimento de intervenções multidisciplinares, de natureza sistêmica e local, nas dimensões de prevenção do agravo, e de suas complicações, bem como de restauração da integridade dos tecidos, com a instalação de terapias apropriadas ao tipo de circulação comprometida;

- organização do serviço para implantação de um sistema de atendimento que permita: abordagem para os grupos específicos, tais como o do presente estudo, que eram predominantemente homens com idade entre 50 a 70 anos, com história de trabalho na construção civil e em atividades rurais, a maior parte da vida, com baixa renda e baixa escolaridade, que não exerciam atividades ocupacionais na vigência das lesões.

\section{CONCLUSÃO}

Os resultados deste estudo corroboram em parte o perfil socioeconômico e demográfico da população acometida por ulceras vasculogênicas, atendida nas instituições públicas de saúde, no contexto ambulatorial. Evidencia-se a baixa renda e baixa escolaridade como característica marcante. Há divergência dos achados desta pesquisa com a literatura, em relação ao gênero que predominou entre a população afetada e quanto à faixa etária de pessoas com úlceras vasculogênicas, que sugere a necessidade de maiores investigações e em diferentes cenários.

Os resultados reforçam a predominância de úlceras venosas entre as vasculogênicas, com apresentação de lesão única de modo mais frequente, de grande extensão, exsudativas, localizada na zona 2 de modo isolado ou combinado com outras zonas. As características do tecido no leito das feridas indicaram comprometimento do processo de cicatrização.

As associações verificadas neste estudo demonstraram a exposição dos indivíduos à limitações funcionais causada pelas más condições das lesões, o que repercute multidimensionalmente.
2. Royal College of Nursing. Clinical Practice Guidelines. The nursing management of patients with venous leg ulcers: recommendations [Internet]. London; 2006 [cited 2010 Mar 2]. Available from: http://www.rcn.org.uk/data/assets/pdf_file/0003/107940/003020.pdf 
3. Etufugh CN, Phillips TJ. Venous ulcers. Clin Dermatol. 2007;25(1):121-30.

4. Koupidis SA, Parakevas KI, Stathopoulos V, Mikhailidis DP. Impact of lower extremity venous ulcers due chronic venous insufficiency on quality of life. Open Cardiovasc Med J. 2008;2:1055-9.

5. Kelechi, T, Bonham, P A. Lower Extremity Venous Disorders: Implications for Nursing Practice. J Cardiovasc Nurs. 2008;23(2):132-43.

6. Edwards $H$, Courtney $M$, Finlayson $K$, Shuter $P$, Lindsay $E$. A randomised controlled trial of a community nursing intervention: improved quality of life and healing for clients with chronic leg ulcers. J Clin Nurs. 2009;18(11):1541-9.

7. Finlayson K; Edwards $\mathrm{H}$; Courtney M. Factors associated with recurrence of venous leg ulcers: a survey and retrospective chart review. Int J Nurs Stud. 2009;46(8):1071-8.

8. Jull A, Parag V, Walker N, Maddison R, Kerse N, Johns T. The prepare pilot RCT of home-based progressive resistance exercises for venous leg ulcers. J Wound Care. 2009;18(12):497-503.

9. Milic DJ, Zivic SS, Bogdanovic DC, Jovanovic MM, Jankovic RJ, Milosevic ZD, et al. The influence of different sub-bandage pressure values on venous leg ulcers healing when treated with compression therapy. J Vasc Surg. 2010;51(3):655-61.

10. Carmo SS, Castro CD, Rios VS, Sarquis MGA. Atualidades na assistência de enfermagem a portadores de úlcera venosa. Rev Eletr Enferm [Internet]. 2007 [citado 2010 abr. 21];9(2): 506-17. Disponível em: http://www.fen.ufg.br/revista/v9/ n2/v9n2a17.htm

11. Conferencia Nacional de Consenso sobre Úlceras de la Extremidad Inferior. Documento de consenso C.O.N.U.E.I. Barcelona: Kamed; 2009.

12. MacGuckin M, Waterman R, Brooks J, Cherry G, Porten $L$, Hurley $S$, et al. Validation of venous leg ulcer guidelines in the United States and United Kingdom. Am J Surg. 2002;183(2):132-7.
13. Heinen $M M$, Persoon $A$, Kerkhof $P$, Otero $M$, Achterberg $T$. Ulcer-related problems and health care needes in patients with venous leg ulceration: a descriptive, cross-sectional study. Int J Nurs Stud. 2006;44(8):1296-303.

14. Bergonse FN, Rivitti EA. Avaliação da circulação arterial pela medida do índice tornozelo/braço em doentes de úlcera venosa crônica. An Bras Derm. 2006;81(2):131-5.

15. Deodato OON. Avaliação da qualidade da assistência aos portadores de úlceras venosas atendidos no ambulatório de um hospital universitário em Natal/RN [dissertação]. Natal: Departamento de Enfermagem, Universidade Federal do Rio Grande do Norte; 2007.

16. Torres GV, Costa IKF, Dantas DV, Dias TYAF, Nunes JP, Deodato OON, et al. Idosos com úlceras venosas atendidos nos níveis primário e terciário: caracterização sociodemográfica, de saúde e assistência. Rev Enferm UFPE. 2009;3(4):222-30.

17. Martins MA. Avaliação de feridas crônicas de pacientes ambulatoriais de Unidades Básicas de Saúde, em Goiânia/GO [dissertação]. Goiânia: Faculdade de Enfermagem, Universidade Federal de Goiás; 2008.

18. Moffatt CJ, Franks PJ, Doherty DC, Martin R, Blewett R, Ross F. Prevalence of leg ulceration in a London population. QJM. 2004;97(7):431-7.

19. Olson JM, Raugi GJ, Nguyen VQ, Yu O, Reiber GE. Guideline concordant venous ulcer care predicts healing in a tertiary care Veterans Affair Medical Center. Wound Repair Regen. 2009;17(5):666-70.

20. Mackenzie RK, Brown DA, Allan PL, Bradbury AW, Ruckley CV. A comparison of patients who developed venous leg ulceration before and after their 50th Birthday. Eur J Vasc Endovasc Surg. 2002;26(1):176-8.

21. Santos VLCG, Sellmer D, Massulo MME. Confiabilidade interobservadores do Pressure Ulcer Scale for Healing (PUSH), em pacientes com úlceras crônicas de perna. Rev Latino Am Enferm. 2007;15(3):391-6.

22. Rossi FR, Silva MAD. Fundamentos para processos gerenciais na prática do cuidado. Rev Esc Enferm USP. 2005;39(4):460-8. 